\title{
Structured Imagery Processing Strategies for Spectroscopic Measurement in Dense Solutions
}

\author{
Thomas Koffi'1,2, Guy-Oscar Regnima1, Jocelyne Bosson², Olivier Bagui', Mensah Edoé1, \\ Jérémie T. Zoueu ${ }^{*}$ \\ ${ }^{1}$ Laboratoire d'Instrumentation, Image et Spectroscopie(L2IS), Institut National Polytechnique Felix Houphouët-Boigny, \\ Departementde Formation et de Recherche du Genie Electrique et Electronique, Yamoussoukro, Côte d'Ivoire \\ ${ }^{2}$ Laboratoire de physique Fondamantale Appliquée(LPFA), Université NanguiAbrogoua, Unité de Formation et de Recherche des \\ Sciences Fondamentales Appliquées, Abidjan, Côte d'Ivoire \\ Email: *Jeremie.zoueu@inphb.ci
}

How to cite this paper: Koffi, T., Regnima, G.-O., Bosson, J., Bagui, O., Edoé, M. and Zoueu1, J.T. (2017) Structured Imagery Processing Strategies for Spectroscopic Measurement in Dense Solutions. Open Journal of Applied Sciences, 7, 262-281. https://doi.org/10.4236/ojapps.2017.76022

Received: April 14, 2017

Accepted: June 16, 2017

Published: June 19, 2017

Copyright $\odot 2017$ by authors and Scientific Research Publishing Inc. This work is licensed under the Creative Commons Attribution International License (CC BY 4.0).

http://creativecommons.org/licenses/by/4.0/

\begin{abstract}
Absorbance measurement in dense media via conventional optical spectroscopy techniques leads to inaccurate results. This is mainly due to multiple scattering phenomena that contribute to the overall light extinction in the interrogated sample. This limitation imposes the use of dilute solutions for absorption spectroscopy. However, depending on the polarity of the solvent used, the absorption spectrum may vary over a given solution. Structured illumination technique offers an alternative to this problem, and provides the ability to calculate in-situ optical properties in dense media. In this paper, we propose two processing methods applied to images acquired by structured laser illumination planar imaging (SLIPI) technique to extract extinction coefficients $\mu_{e}$ of probed solutions: The first is based on the implementation of principal component analysis (PCA) and the second, on the calculation of Mean Value. In practice, two kinds of studies were carried out: one quantitative set of measurements within chlorophyll liquid solutions and a second set with concentrated coffee solutions, with controlled proportion and concentrations for each sample. These two proposed analytical techniques are advantageous because they are very easy to implement and provide a much simpler alternative to the previous one. Both methods offer satisfactory results, similar to those obtained with the original method which is based on 1D Fourier transform.
\end{abstract}

\section{Keywords}

Spectrophotometry, Dense Media, Extinction Coefficient, Structured Illumination, Principal Component Analysis

\section{Introduction}

Matter is mostly made up of structures with various properties that react in var- 
ious ways to photon interaction. This is the case, for example, of biological tissues whose composition is inhomogeneous and sometimes dense. This, in homogeneity property of the matter, becomes a challenging issue in diagnosis using classical method of optical spectroscopy. It uses the Beer-Lambert law which establishes the proportionality between the concentration of chemical species, the absorptivity and the path length traveled by the light in the media [1] [2] [3] [4]. However, this law is no more applicable while measuring the absorbance of media for which transmittance is either very high, or too weak, under certain conditions. Thus, there are important operational conditions under which the Beer-Lambert law cannot be applied without modification [2] [4] [5] [6] [7] [8]. So, to overcome these physical limitations and making successful spectroscopic measurements in turbid environments, a relatively new approach based on structured illumination was developed by Edouard Berrocal and coworkers [9]. This technique named SLIPI (Structured Illumination Laser Planar Imaging) is based on the use of spatially modulated laser sheet passing through the studied dense media. The light pattern formed inside the media is captured and processed to get rid of the multiple scattering photons. The main idea behind using the spatial modulation of the incident light intensity is that only the ballistic photons will keep the memory of the light pattern and lead to significant improvement of contrast of images [10]. The stray light is extracted and is used to calculate the optical density [11]. This approach has been successfully used for 3D imaging and characterizing of scattering media [12]. Kristensson and coworkers have also used the same idea in the context of imaging of molar extinction coefficients of turbid media [13]. And most recently Olivier K. Bagui et al have used it for classifying groups of coffee [14].

In this work, we have developed new data processing techniques applied to the same types of images acquired by SLIPI technique. These analytical methods are based on algorithms edited in our laboratory, based on Principal Component Analysis (PCA) method, and Mean Value (MV) method. Both methods provide satisfactory and interesting results compared to the previous method.

In practice, we have measured firstly the extinction coefficients of several chlorophyll dense solutions, extracted from fresh banana leaves. The use of chlorophyll as a sample would be beneficial in preventing food shortages and protecting vegetation. This could lead to a study of vegetation protection against harmful factors of climate change. Secondly, we applied these proposed methods to dense liquid coffee solutions whose concentrations are very well known and whose mode of preparation is well defined. Note that the results obtained from these three methods, for each sample examined with laser radiation of wavelengths $450 \mathrm{~nm}$ and $638 \mathrm{~nm}$, have shown the pertinence of our computational algorithms, because they provide similar results to the existing method. They are also rather fast and provide very similar results to those of the existing method. The evaluated variations between extinction coefficients calculated via the standard method and those that we propose are estimated between $0.00 \%$ and $2.13 \%$ which appear very weak. Such observations could confirm the relevance of the 
suggested techniques with a minimal risk of error.

\section{Principle of SLIPI Technique}

The SLIPI technique was inspired by structured illumination microscopy principle [15]. SLIPI method combines both structured illumination and laser planar imaging by superimposing a periodic pattern with a laser sheet [16]. The main idea is to filter the single scattered photons from multiple scattered lights by assuming that the photons satisfying the Beer-Lambert law have undergone a single diffusion before reaching the detector. For classical spectroscopy, this equation remains valid when the particle density is low, and when the path length traveled by all photons in the examined sample is short. However, SLIPI technique is based on a principle that allows the selective encoding of ballistic photons that will keep the memory of the spatial modulated pattern even in turbid media, and to consider the multiple scattered photons as noise (whose magnitude depends on the optical characteristics of the sample) [17]. This process works as directional filter and extract most of the unique diffusion of photons in the propagation direction, considerably improving the contrast of the image [18]. Note that the principle of this technique is entirely well described in the literature [19] [20] [21] [22], particularly in Chapter 6 of Kristensson PhD Thesis (see Ref. [18]). An image obtained from a sample illuminated by an intensity modulated with a sinusoidal pattern is described by:

$$
I(x, y)=I_{c}(x, y)+I_{s}(x, y) \cos (2 \pi x v+\varphi)
$$

where $v$ is the modulation frequency, $\varphi$ is the spatial phase $I_{c}(x, y)$, the intensity of the single scattering and multiple scattering, and $I_{s}(x, y)$ is the amplitude of the single scattering. The SLIPI technique allows to extract and preserve $I_{s}(x, y)$ while it removes $I_{c}(x, y)$ and $\cos (2 \pi x v+\varphi)$ image after processing. To extract the single scattering information and to get the SLIPI image $I_{s}(x, y)$ experimentally, a minimum of three intensity-modulated images are recorded with a spatial phase corresponding to $0^{\circ}, 120^{\circ}$ and $240^{\circ}$. By calculating the pairwise difference between the three recorded images, most of the multiple scattering of light is suppressed. Equations ((2) and (3)) show how SLIPI image and the conventional image can be extracted from three images spatially modulated [23].

$$
\begin{gathered}
I_{s}=\frac{\sqrt{2}}{3}\left[\left(I_{0}-I_{120}\right)^{2}+\left(I_{0}-I_{240}\right)^{2}+\left(I_{120}-I_{240}\right)^{2}\right]^{\frac{1}{2}} \\
I_{c}=\frac{I_{0}+I_{120}+I_{240}}{3}
\end{gathered}
$$

By registering $N$ spatially modulated images with a relative phase shift of $\frac{2 \pi}{N}$, the SLIPI image can be formed from the generalized Equation (4).

$$
I_{s}=\frac{\sqrt{2}}{N}\left(\sum_{i=1}^{N-1} \sum_{j=i+1}^{N}\left(I_{i}-I_{j}\right)^{2}\right)^{\frac{1}{2}}, \quad N \geq 3
$$




\section{Materials and Methods}

\subsection{Samples Acquisition}

\subsubsection{Chlorophyll Solutions}

The first examined media were chlorophyll solutions. The chlorophyll solutions were exclusively extracted from fresh leaves of five banana trees grown on different sites. These leaves were mashed and infused into alcohol, hence used as a solvent. In order to avoid any unwanted reaction, we proceeded then to the filtering of the obtained solutions, in absence of light. This allowed us to obtain dense solutions of chlorophyll from fresh leaves of each plant noted $S_{1}, S_{2}, S_{3}, S_{4}$ and $S_{5}$. These solutions were classified according to their level of turbidity: From high-concentrated $\left(S_{1}\right)$ to lowest $\left(S_{5}\right)$.

\subsubsection{Coffee Solutions}

The coffee solutions are prepared using instant coffee (from the brand "Nescafé Classic") made of $100 \%$ robust a coffee. An initial solution $\mathrm{C}_{1}$ is created by dissolving 6 grams of coffee into $450 \mathrm{ml}$ of water. This dilution results to a concentration equals to $1 \%$ from the initial coffee concentration $\mathrm{C}_{1}$. From this solution, 4 solutions have been, once again, prepared to obtain a linear decrease in concentration.

\subsection{Experimental Setup}

The SLIPI technique used in solving optimization problems in the fuel efficiency, had proven to be capable of multiple scattering suppressing in dense sprays imaging [9]. And since then, according to the assigned objective, its experimental device is available in various configurations. We use the SLIPI single-phase approach whose schematic representation can be seen in Figure 1, to measure the extinction coefficient of probed dense liquids samples. In practice, a sample placed in a silica cuvette is successively illuminated by radiation from two lasers diode of $450 \mathrm{~nm}$ and $638 \mathrm{~nm}$. These laser beams are expanded and collimated through two spherical lenses placed one after the order in such a way that the

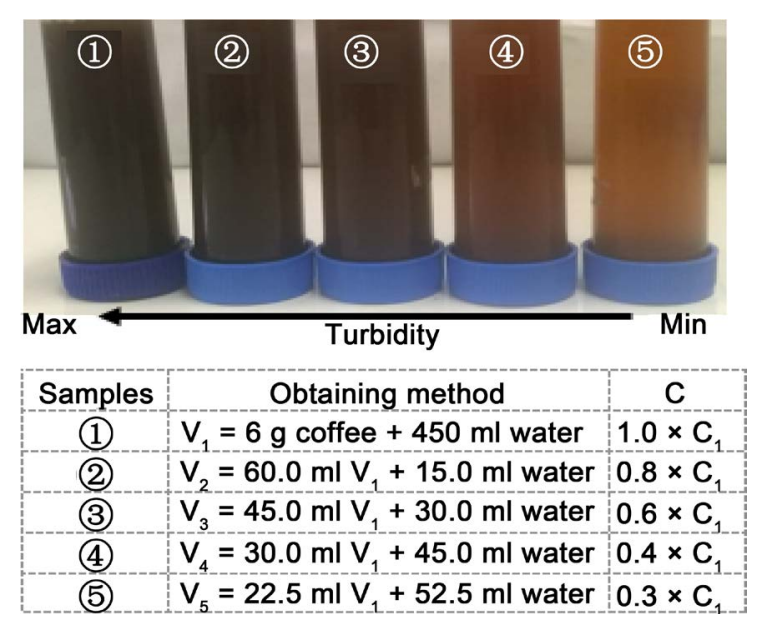

Figure 1. Samples of coffee solutions photographed in top and the corresponding dilution procedure is provided in the table below. 


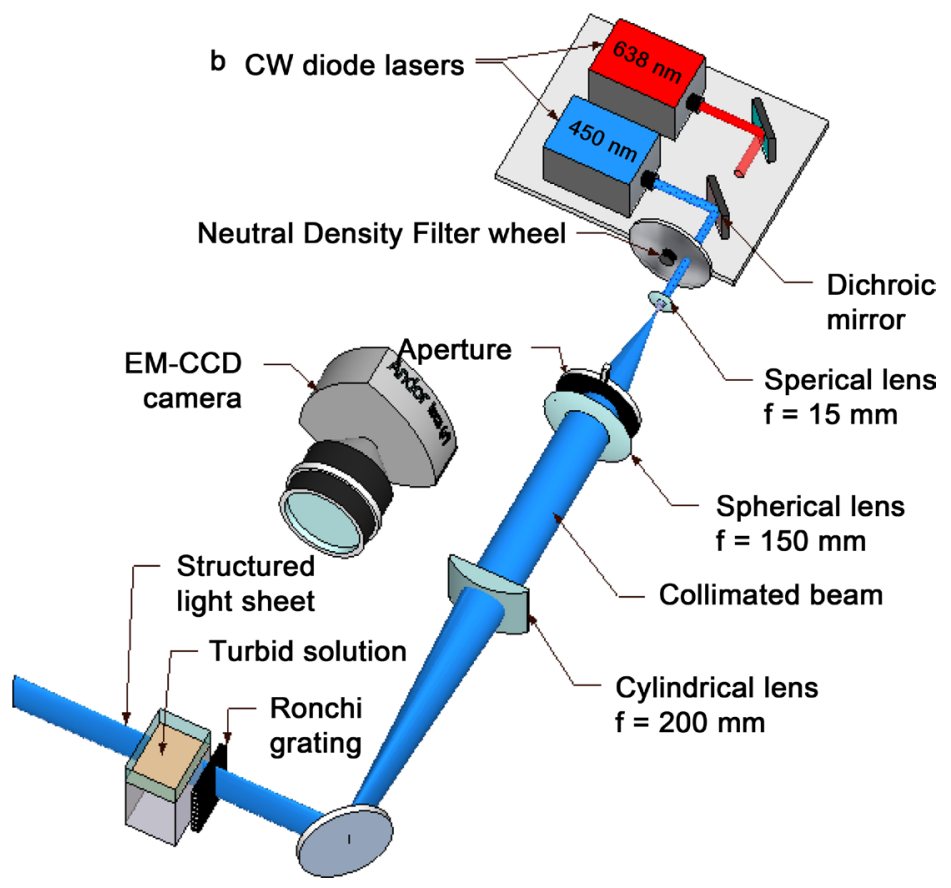

Figure 2. A detailed schematic of single-phase SLIPI optical arrangement.

distance between them is equal to the sum of these focal lengths. The laser sheet created by a cylindrical lens, and modulated using a $5 \mathrm{lp} / \mathrm{mm}$ Ronchi grating is then completely reflected through a plane mirror on the cuvette containing the sample. A 14-bit EM-CCD camera (maximum number of photons $2^{14}-1=$ 16383) located at $90^{\circ}$ to the propagation direction of the laser light sheet, capture the modulated image generated in the cuvette. The recording of acquired images is provided by a personal computer linked to the camera (see Figure 2).

\subsection{Analysis Methods}

\subsubsection{The Single-Phase SLIPI Approach}

The standard strategies used in order to process the images acquired by SLIPI set-up and applied to turbid media, is based on single phase scattering detection by means of Fourier transform [9]. The so-called lock-in detection method is widely developed in Ref [10]. The explanation of the spatial lock-in algorithm mathematically, leads to consider a column vector in the modulated spectrum (I) at the wavelength $\lambda$ containing a spectral line:

$$
I(\lambda, y)=A_{\lambda}(y) \sin \left(2 \pi v_{g} y+\phi_{\lambda}(y)\right)+B_{\lambda}(y)
$$

where $y$ is the spatial vector, $v_{g}$ is the the grating modulation frequency, $A$ represents the amplitude of the modulated signal, $B$ a non-modulated background, and $\phi_{\lambda}(y)$ the unknown spatial phase of the superimposed modulation. The extraction of $A$ is main goal of lock-in algorithm. Then, extract $A$ and reject $B$.

For that purpose, one creates two reference signals $I_{r 1}$ and $I_{r 2}$ with identical period $\left(v_{g}\right)$ which is equal to those of the modulated spectrum according to the following Equations ((6) and (7)) but phase-shifted $\pi / 2$ [24]. 


$$
\begin{gathered}
I_{r 1}=\sin \left(2 \pi v_{g} y\right) \\
I_{r 2}=\cos \left(2 \pi v_{g} y\right)
\end{gathered}
$$

Multiplying the column vector $I(\lambda, y)$ with the reference in Equation 5, signals yields:

$$
I_{\lambda}^{1}=I_{r 1} I(\lambda, y)=A_{\lambda} \sin \left(2 \pi v_{g} y+\phi_{\lambda}(y)\right) \sin \left(2 \pi v_{g} y\right)+B_{\lambda}(y) \sin \left(2 \pi v_{g} y\right)
$$

And

$$
I_{\lambda}^{2}=I_{r 2} I(\lambda, y)=A_{\lambda} \sin \left(2 \pi v_{g} y+\phi_{\lambda}(y)\right) \cos \left(2 \pi v_{g} y\right)+B_{\lambda}(y) \cos \left(2 \pi v_{g} y\right)
$$

Which can be simplified to

$$
I_{\lambda}^{1}=\frac{1}{2} A_{\lambda}\left(\cos \left(\phi_{\lambda}(y)\right)-\cos \left(4 \pi v_{g} y+\phi_{\lambda}(y)\right)\right)+B_{\lambda} \sin \left(2 \pi v_{g} y\right)
$$

And

$$
I_{\lambda}^{2}=\frac{1}{2} A_{\lambda}\left(\sin \left(\phi_{\lambda}(y)\right)-\sin \left(4 \pi v_{g} y+\phi_{\lambda}(y)\right)\right)+B_{\lambda} \cos \left(2 \pi v_{g} y\right)
$$

Three components can be identified by frequency analyzing of $I_{\lambda}^{1}$ and $I_{\lambda}^{2}$ in the two preceding Equation (8) and Equation (9). First, (a) a DC component, then (b) one modulated with $2 v_{g}$ and finally (c) one modulated with $v_{g}$. It is now advisable to remove the two last components in the Fourier domain applying a low-pass filter having a cut-off frequency $\left(v_{c}\right)$ less than $v_{g}$, resulting in the following expressions:

$$
\begin{aligned}
& \tilde{I}_{\lambda}^{1}=\frac{1}{2} \tilde{A}_{\lambda} \cos \left(\phi_{\lambda}(y)\right) \\
& \tilde{I}_{\lambda}^{2}=\frac{1}{2} \tilde{A}_{\lambda} \sin \left(\phi_{\lambda}(y)\right)
\end{aligned}
$$

The tilde assignment in these relations indicates the applied frequency filtering. From these, $\tilde{A}_{\lambda}$ can be extracted finally thanks to the following formula [24]:

$$
\tilde{A}_{\lambda}=2 \sqrt{\left(\tilde{I}_{\lambda}^{1}\right)^{2}+\left(\tilde{I}_{\lambda}^{2}\right)^{2}}
$$

One can extract the amplitude of the modulation from a modulated image using Equations (6)-(14). The modulated component $A_{\lambda}$, reflects the exponential decay of the single-scattered light along the cross-sectional width of the cuvette. Extracting the extinction coefficient $\mu_{\mathrm{e}}$ can be done by applying an exponential fit to $A_{\lambda}$ according to the Beer-Lambert-Bouguer absorption law.

Figure 3 and Figure 4 show illustrations of the single-phase SLIPI process.

The amplitude of the intensity decreases from column $\mathrm{P}$ to $\mathrm{Q}$ according to the light intensity profile represented. The 1D Fourier transform (FT) applied to the curves $\mathrm{P}$ and $\mathrm{Q}$, makes it possible to obtain the reduction in strength of the $1^{\text {st }}$ order peak (modulation frequency). This frequency is then filtering and isolated after applying the lock-in algorithm, which finally reveals the exponential decay (see Figure 4). 


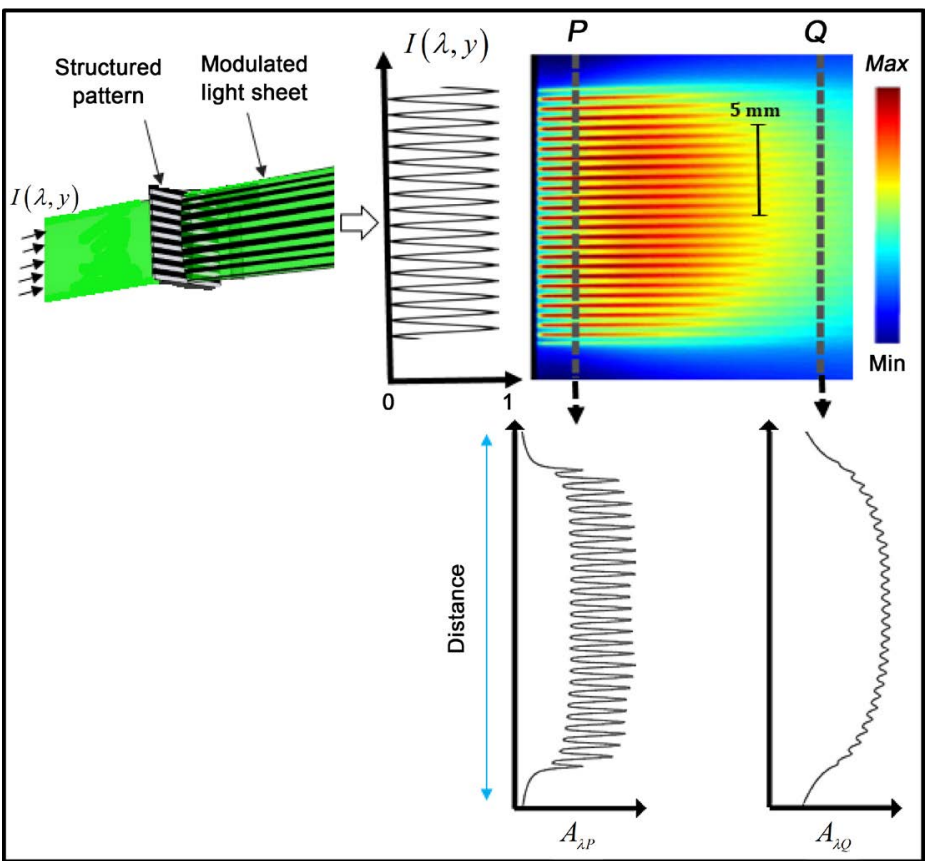

Figure 3. Single-phase SLIPI principle. This illustration represents the signal from a structured laser sheet, with cross-sections extracted from two different depths, marked as $\mathrm{P}$ and $\mathrm{Q}$.

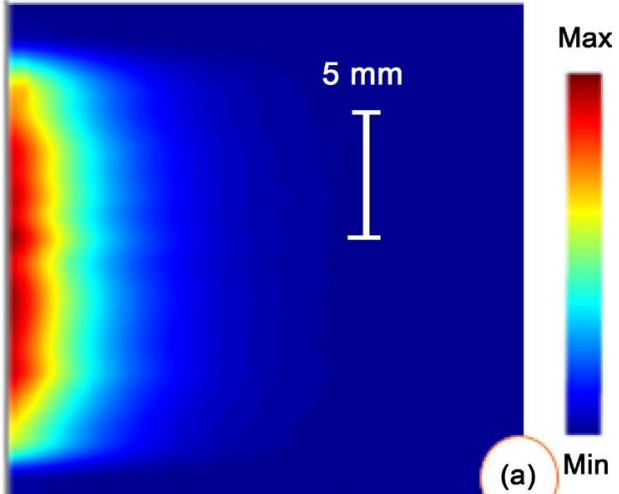

(a)

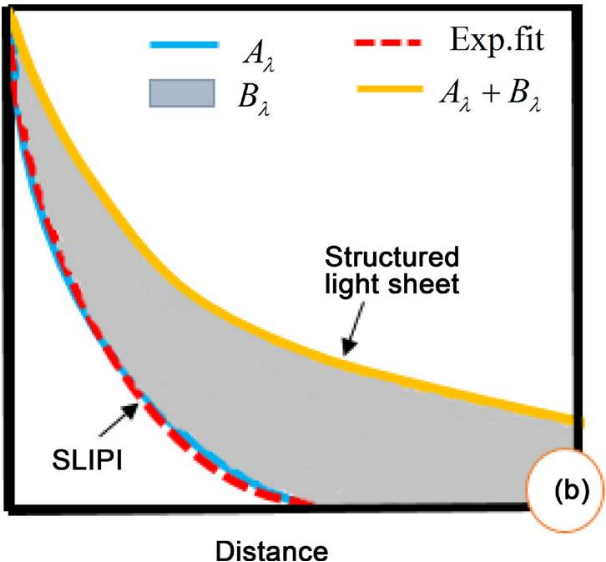

Figure 4. (a) SLIPI image. Isolated frequency using frequency filtering obtained by means of lock-in algorithm. (b) Curves corresponding to the exponential decay of the light intensity crossing the sample after filtering. 


\subsubsection{Mean Value and PCA Approach}

The analysis methods we are proposing are applied to the images captured by the CCD camera, produced from the convolution of the diffracting pattern by the system's transfer function. These analysis techniques are based on algorithms developed using Matlab R2014a; and globally aim at filtering the light intensity crossing the solution, in order to evaluate and extract noises. We make the assumption that the light passing through the studied media is composed of two components: The multiple scattered photons which are uncorrelated along the optical path and then regarded as noises, and the single scattered light possessing the distinguishing characteristics imposed by the spatial modulation. These codes based on a signal processing have a principle stipulating that the sum of intensities measured on each line of the diffraction pattern, plus the sum of the noises must be equal to an intensity that will tend to the incident flux $I_{0}$ according to the following relationship 15.

$$
\sum_{n=1}^{\infty} \sum_{i=1}^{\infty}\left[I_{n}+\left(b_{i}\right)_{n}\right] \rightarrow I_{0}
$$

where $\left(b_{i}\right)_{n}$ is the fluctuating or variable noise which are recorded for each $n$ line, and $I_{n}$ is the recorded intensity on each $n$ line without noise. Let us note that two approaches are developed here; the Mean Value Method and the Principal Component Analysis method.

In the Mean Value Method, we made the average of illuminated lines intensities in the modulation before tracing the total decrease of luminous flow. Therefore, the hypothesis that emanates from this approach is that, when we make the average line by line, the mean value of the intensity obtained will tend towards $I_{0}$ which is considered as the single scattered light.

In the Principal Component Analysis (PCA) method, we extract the correlations between lines. This method consists of applying principal component analysis on each line. Therefore, the same assumption: $I_{n}$ will be added with a noise in each line:

$I_{1}+\sum_{i}\left(b_{i}\right)_{1}$ (1 $1^{\text {rst }}$ line $), I_{2}+\sum_{i}\left(b_{i}\right)_{2},\left(2^{\text {nd }}\right.$ line $), \ldots, I_{n}+\sum_{i}\left(b_{i}\right)_{n}, n^{\text {th }}$ line, assuming $I_{n}$ on each $n$ line does not vary, as shown in Figure 5 .

Thus, by extracting the correlations existing between the lines, the first principal component that we will obtain, must correspond to $I_{1}$ and thus easily dissociable from the noises.

Let us develop now mathematical descriptions which underlie the suggested techniques.

$$
\begin{gathered}
I(x, y)=I_{c}(x, y)+I_{s}(x, y) \cos (2 \pi x v+\varphi) \\
I\left(x_{n}, y\right)=I_{c}\left(x_{n}, y\right)+I_{s}\left(x_{n}, y\right) \cos \left(2 \pi x_{n} v+\varphi\right) \\
\sum_{n=1}^{N} I\left(x_{n}, y\right)=\sum_{n=1}^{N}\left[I_{c}\left(x_{n}, y\right)+I_{s}\left(x_{n}, y\right) \cos \left(2 \pi x_{n} v+\varphi\right)\right] \\
\sum_{n=1}^{N} I\left(x_{n}, y\right)=\sum_{n=1}^{N} I_{c}\left(x_{n}, y\right)+\sum_{n=1}^{N} I_{s}\left(x_{n}, y\right) \cos \left(2 \pi x_{n} v+\varphi\right)
\end{gathered}
$$




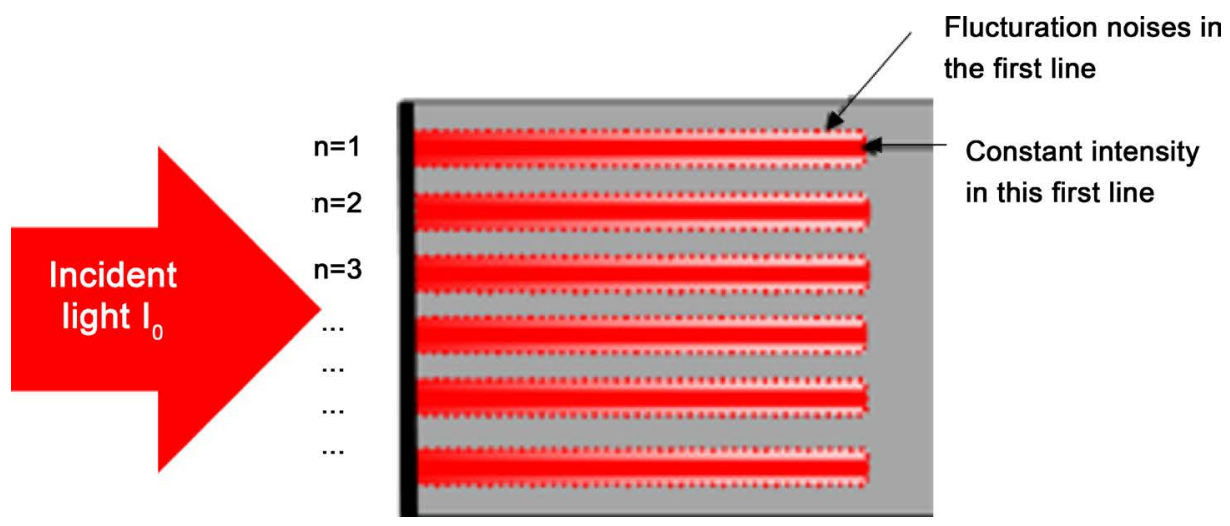

Figure 5. Corresponding diagram to the intensity of light recorded on each line and the associated noises. When the light penetrates in-depth of the liquid solution, the line of the grating becomes less perceptible because of the multiplication of the intensity noise which appears.

On each enlightened line $\mathrm{L}$, the incident light will undergo an exponential decay according to the following equation.

$$
I(L)=I(0) \cdot \mathrm{e}^{\left(-\mu_{e} L\right)}
$$

where $\mu_{e}$ the extinction coefficient.

Let assume that the line pixel is composed of the single scattered photon $J_{S}$ and the multiple scattered photon $J_{M}$.

$J_{S}$ is a function of $y\left(J_{S}=J_{S}(y)\right)$, and $J_{M}$ has a random distribution and doesn't depend on $x$ or $y$.

So let write that:

$$
\begin{gathered}
I\left(x_{n}, y\right)=J_{S}(y)+J_{M} \\
I_{C}\left(x_{n}, y\right)=J_{C S}(y)+J_{C M} \\
I_{S}\left(x_{n}, y\right)=J_{S S}(y)+J_{S M}
\end{gathered}
$$

For all $N$ lines of the diffraction pattern, one can write:

$$
\begin{aligned}
& \sum_{n=1}^{N} I\left(x_{n}, y\right)=\sum_{n=1}^{N}\left[J_{S}(y)+J_{M}\right] \\
& \sum_{n=1}^{N} I\left(x_{n}, y\right)=\sum_{n=1}^{N} J_{S}(y)+\sum_{n=1}^{N} J_{M}
\end{aligned}
$$

As $\mathrm{N}$ increase, $\sum_{n=1}^{N} J_{M}$ tend to zero.

$$
\sum_{n=1}^{N} J_{M} \rightarrow 0
$$

A pre-processing of the raw data consists in extracting the correlations existing between the illuminated lines in the captured image by the CCD. This makes it possible to improve the contrast of the image containing the information on the optical properties of the sampled medium. This technique makes it possible to make the diffracted lines of the grating in the raw image (Figure 6(a)) more perceptible (Figure 6(b)).

Applying Principal Component Analysis (CPA), we obtain the matrix of eigenvalues to target the area of interest containing mainly the information. This 

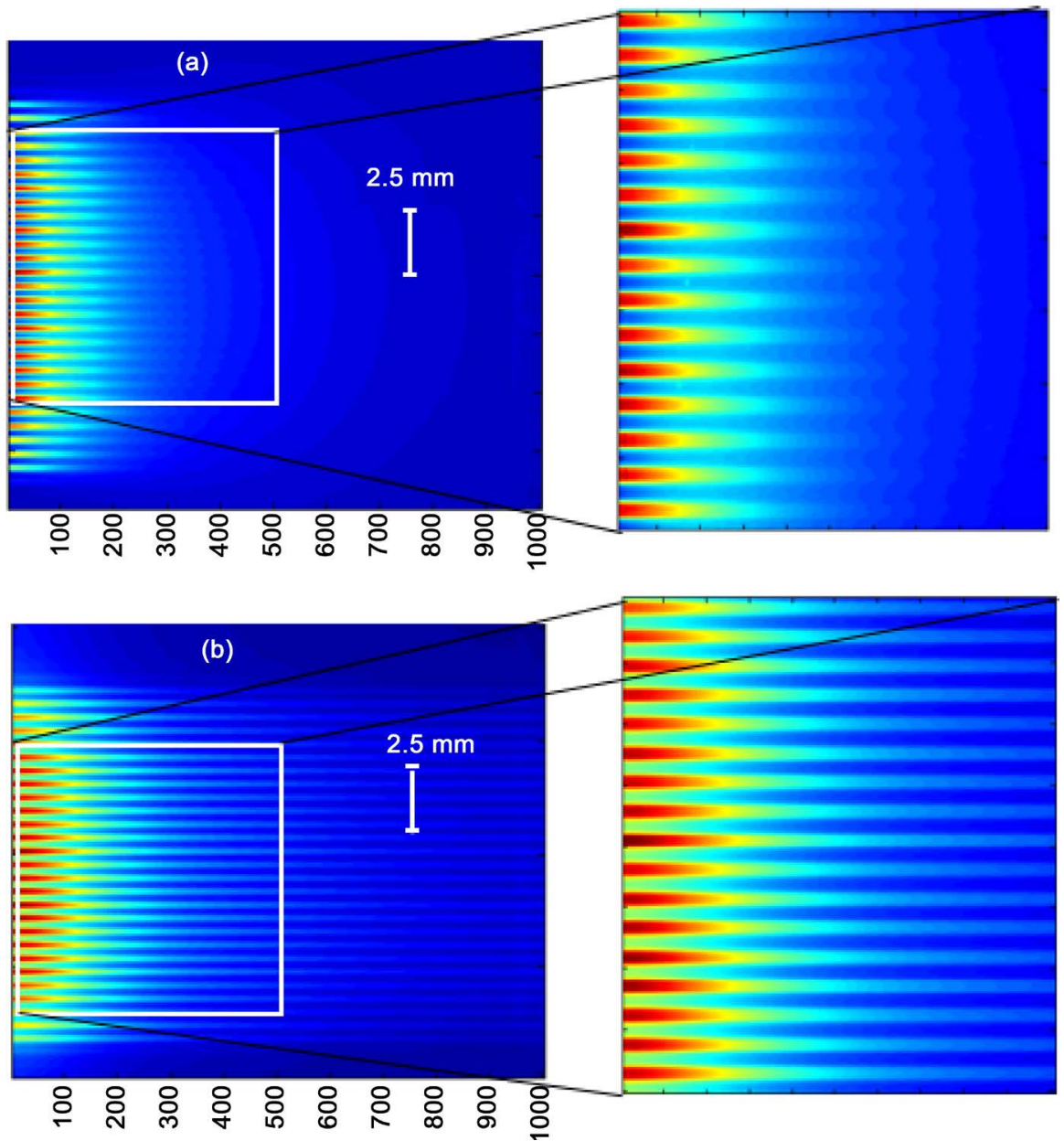

Figure 6. (a) Raw image captured by the CCD. In this image, we can notice that the light diffracted by the grating is only perceptible over a distance approximately equal to 10 $\mathrm{mm}$. (b) Image (a) whose contrast has been enhanced, appearing clearer. In this image, the lines of the diffraction pattern are perceptible over the entire width of the imaged cuvette $(20 \mathrm{~mm})$. Zoom on area of $10 \mathrm{~mm}^{2}$ of the two images are also given.

area corresponds to the more intense intensity part (red parts in Figure 6).

By decomposition into singular values, one succeeds in extracting the principal components, above the red line (Figure 7), which are dissociated to the noises.

All these components then made it possible to reconstitute the matrix making it possible to select the main zone of interest, where the information on the extinction coefficients is more perceived.

In order to understand how the radiation inside the liquid solution could vary, we plotted the histogram of the recorded image (Figure 8).

The histogram clearly shows an exponential decay of the light intensity in the probed sample. This exponential decrease is due to the extinction of light flow, caused both the absorption and diffusion according to the following expressions [18]:

$$
I_{t}=I_{0} \mathrm{e}^{-\left(\mu_{a}+\mu_{s}\right) l}
$$

And 


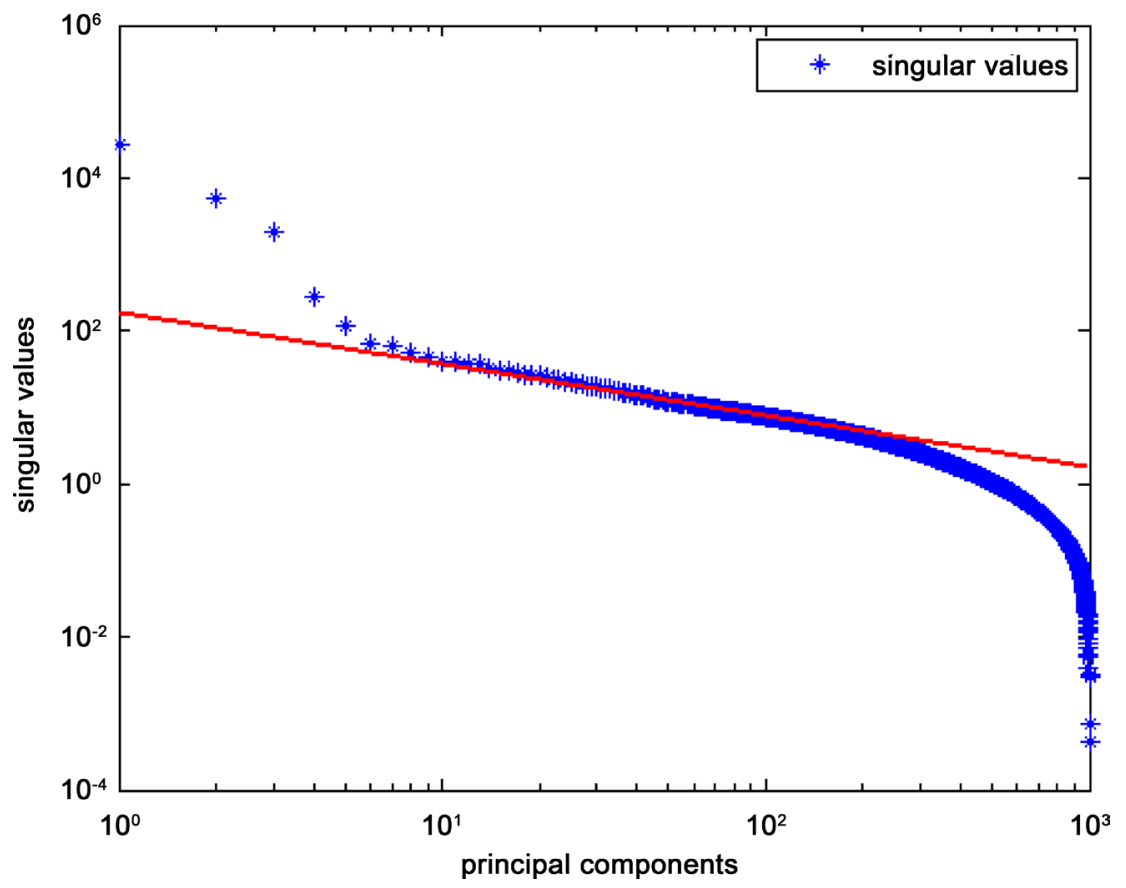

Figure 7. Decomposition into singular values of matrix components and extraction of the main diagonal elements. This can significantly separate the noise and main components. These main eigenvalues which are above the line serve to reconstitute the image which appears clearer.

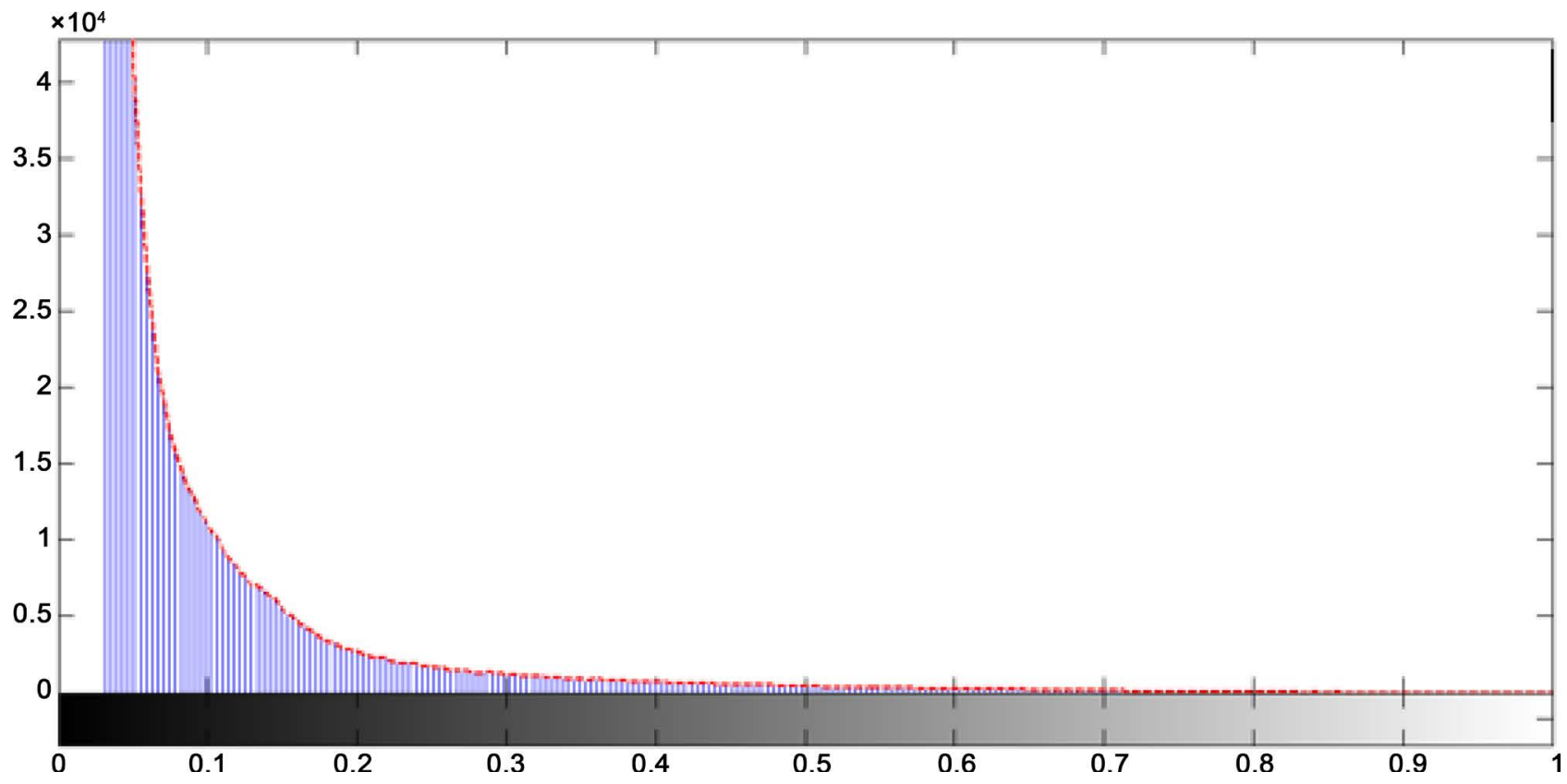

Figure 8. The histogram corresponding to the recorded image. This shows an exponential decrease of the intensity distribution in the recorded image along the $\mathrm{x}$-axis.

$$
\mu_{a}+\mu_{s}=\mu_{e}
$$

where $\mu_{e}, \mu_{a}$ and $\mu_{s}$ are respectively the extinction, absorption and scattering coefficients, $l$ the length of scattering medium. $I_{t}$ is the intensity of transmitted light, $I_{0}$ the incident light intensity. The extinction coefficient de- 
pends, in this case, on the solution density $N$, and on the cross section $\sigma_{e}$ of the extinction. It can be estimated as in the Equation (10) [25].

$$
\mu_{e}=N \cdot \sigma_{e}
$$

Then we plot curves corresponding to the exponential decay of the light intensity crossing the sample after filtering using Mean Value and PCA methods. The extinction coefficients of each solution probed with wavelengths of $450 \mathrm{~nm}$ and $638 \mathrm{~nm}$ can be calculated. These extinction coefficients values are extracted by finding the best exponential fit which can be relatively well superimposed to experimental one, as shown in Figure 9

Then, we look for the convergence of the proposed methods to the standard method.

\section{Results and Discussions}

For each probed sample at $450 \mathrm{~nm}$ and $638 \mathrm{~nm}$, the data processing by each of the three techniques of analysis made it possible to plot the various spectra and to calculate the extinction coefficients. Figures 10-13 show the respective curves resulting from the exponential decay of the light crossing each solution and plotted via each method. The various extinction coefficients values are also calculated using each of the three methods. Those are posted in legend.

For the all examined samples, we calculated and extracted the various extinction coefficients values. All these values are recapitulated in each illustration. Note that chlorophyll solutions extinction coefficients calculated at $450 \mathrm{~nm}$ by the Fourier transform method are all nearly identical to those obtained with the two other proposed methods. The same remark is also made with wavelength $638 \mathrm{~nm}$ for these samples. The extinction coefficients of coffee solutions ob-

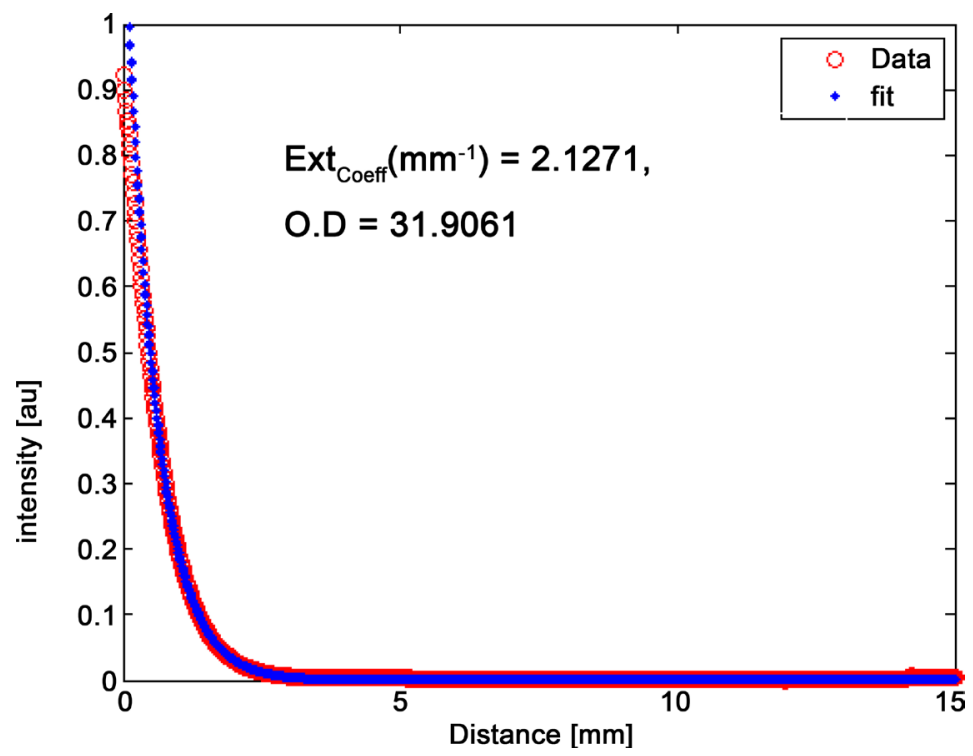

Figure 9. An example of curves obtained via mean value method of the coffee solution $S_{1}$ for $\lambda=450 \mathrm{~nm}$. The values of the extinction coefficient and optical density are posted. It is noticed that the experimental curve (blue) and the theoretical curve (red) are well superimposed. 


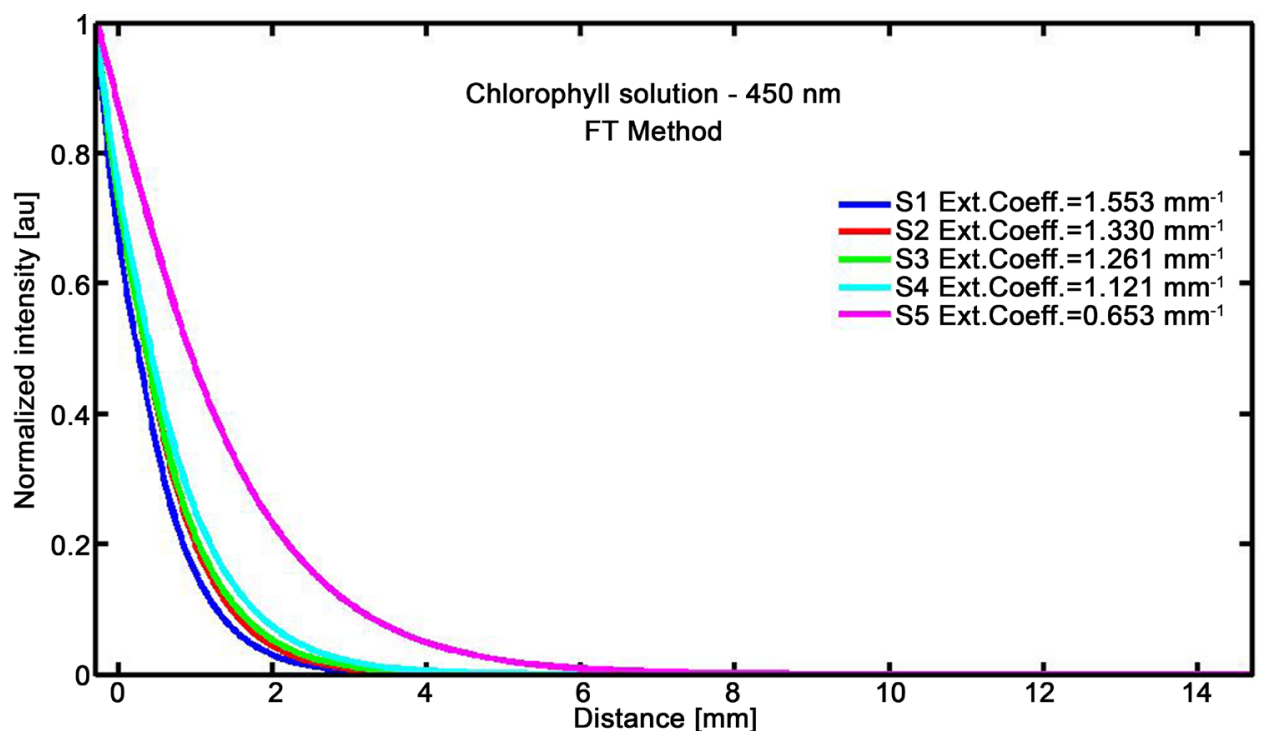

(a)

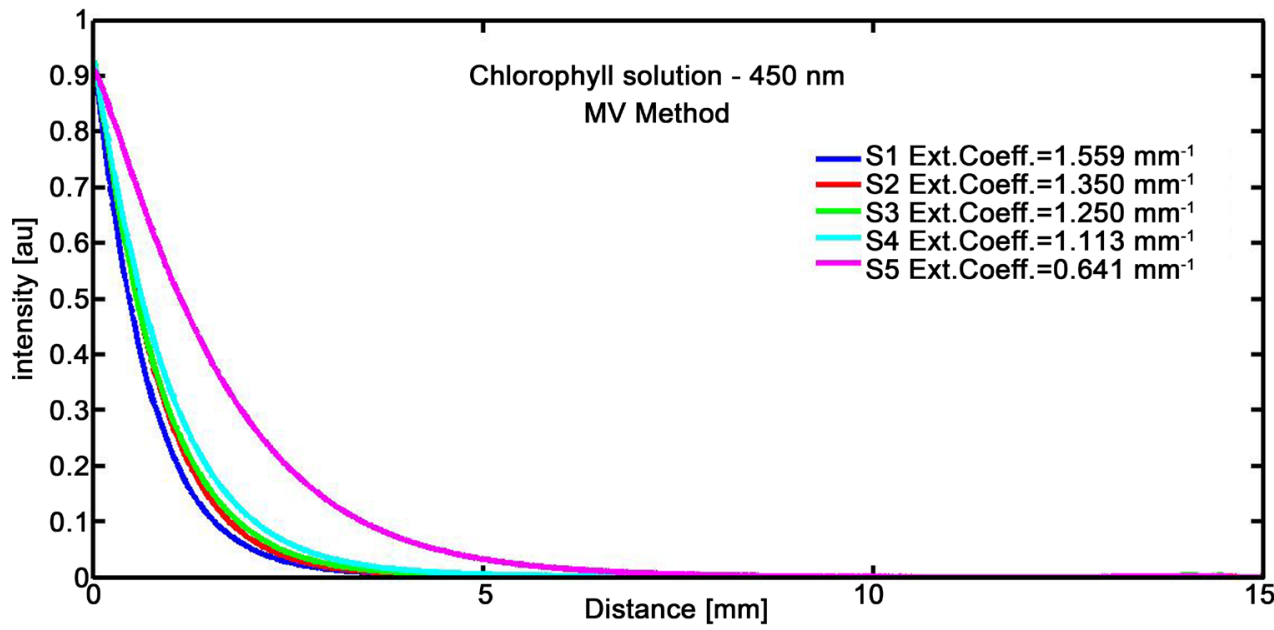

(b)

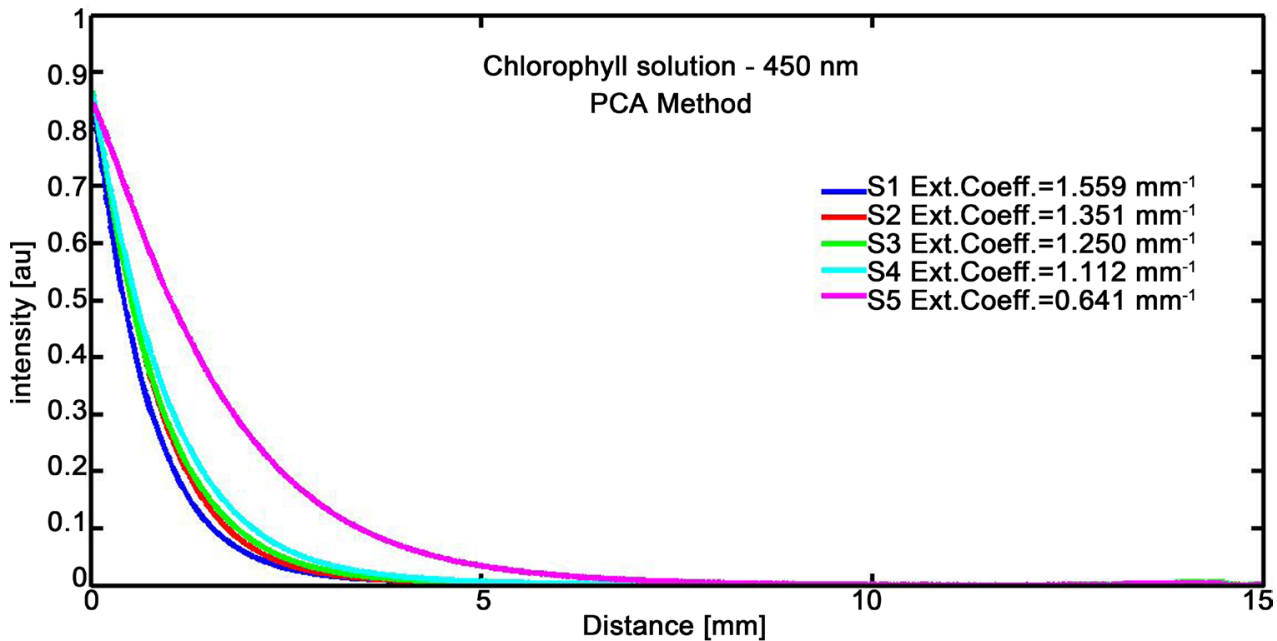

(c)

Figure 10. Curves resulting from the exponential decay of light intensity traced using each of the three methods, for the five samples of chlorophyll solutions probed with laser light at $450 \mathrm{~nm}$. The extinction coefficients values obtained by each method are posted on each illustration. We have in top, the standard method (FT) resulting results; in the medium, those of the Mean Value (MV) method and below, the results resulting from the Principal Components Analyze (PCA) method. 


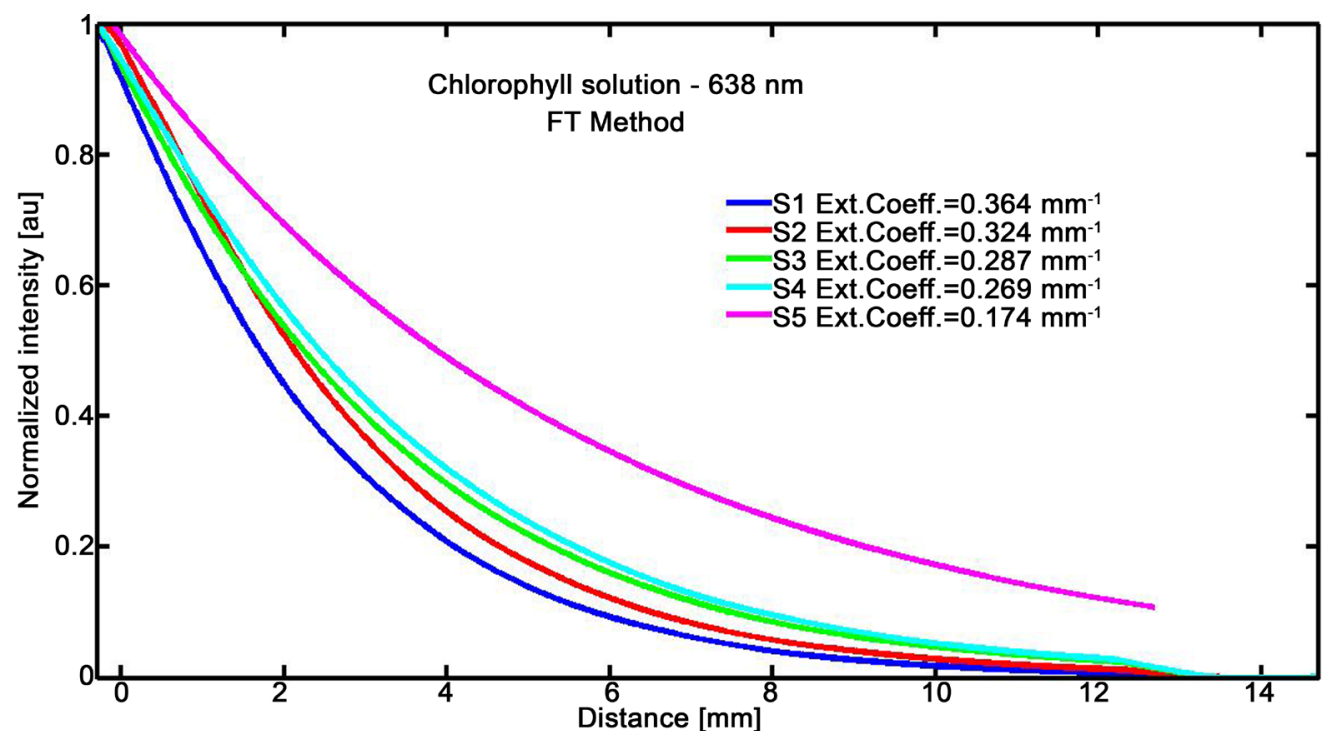

(a)

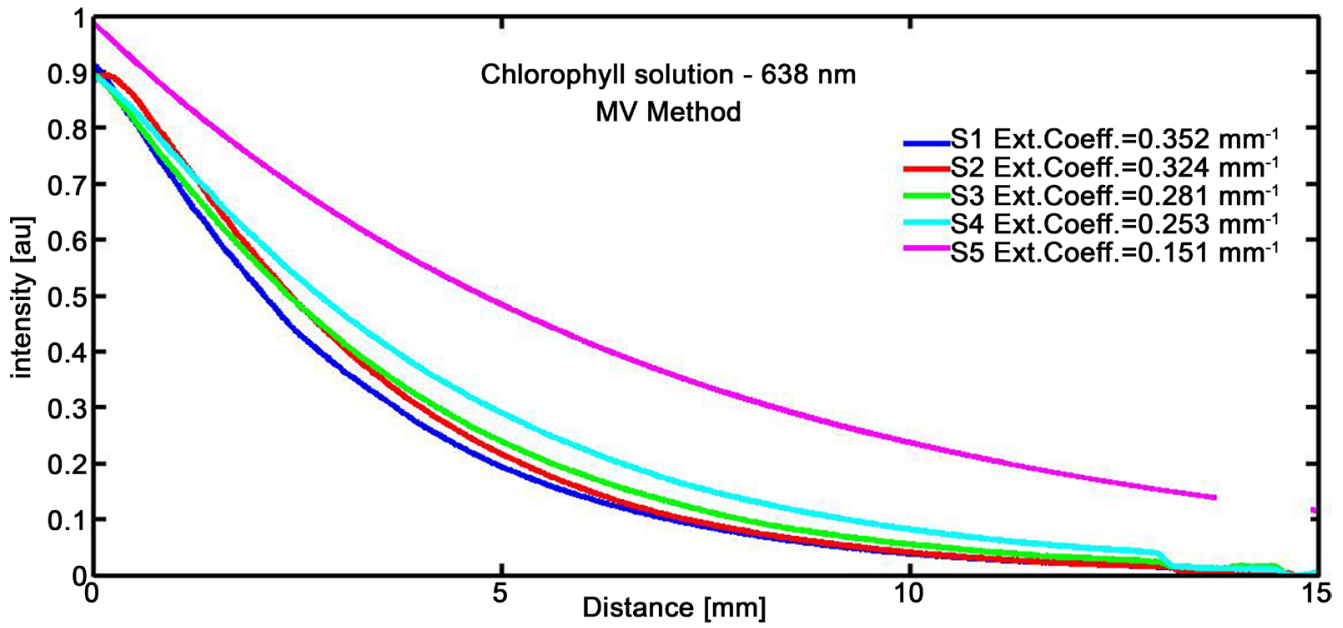

(b)

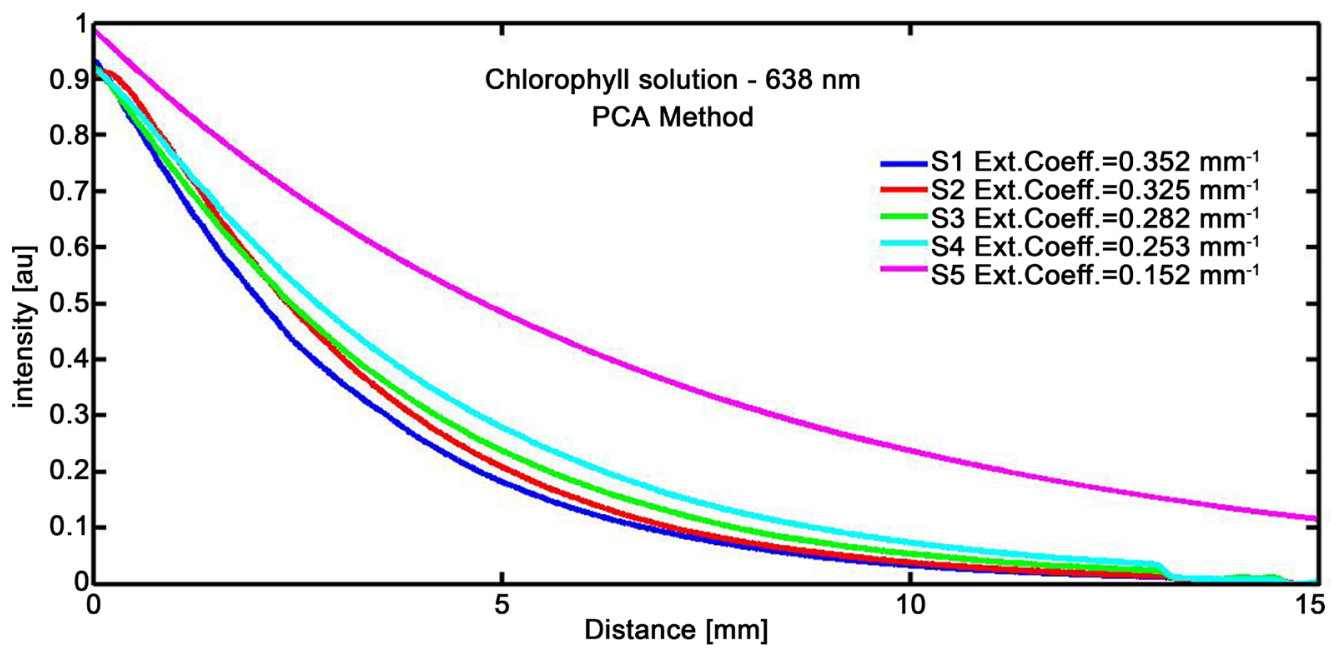

(c)

Figure 11. Curves resulting from the exponential decay of light intensity traced using each of the three methods, for the five samples of chlorophyll solutions probed with laser light at $638 \mathrm{~nm}$. The extinction coefficients values obtained by each method are posted on each illustration. We have in top, the standard method (FT) resulting results; in the medium, those of the Mean Value (MV) method and below, the results resulting from the Principal Components Analyze (PCA) method. 


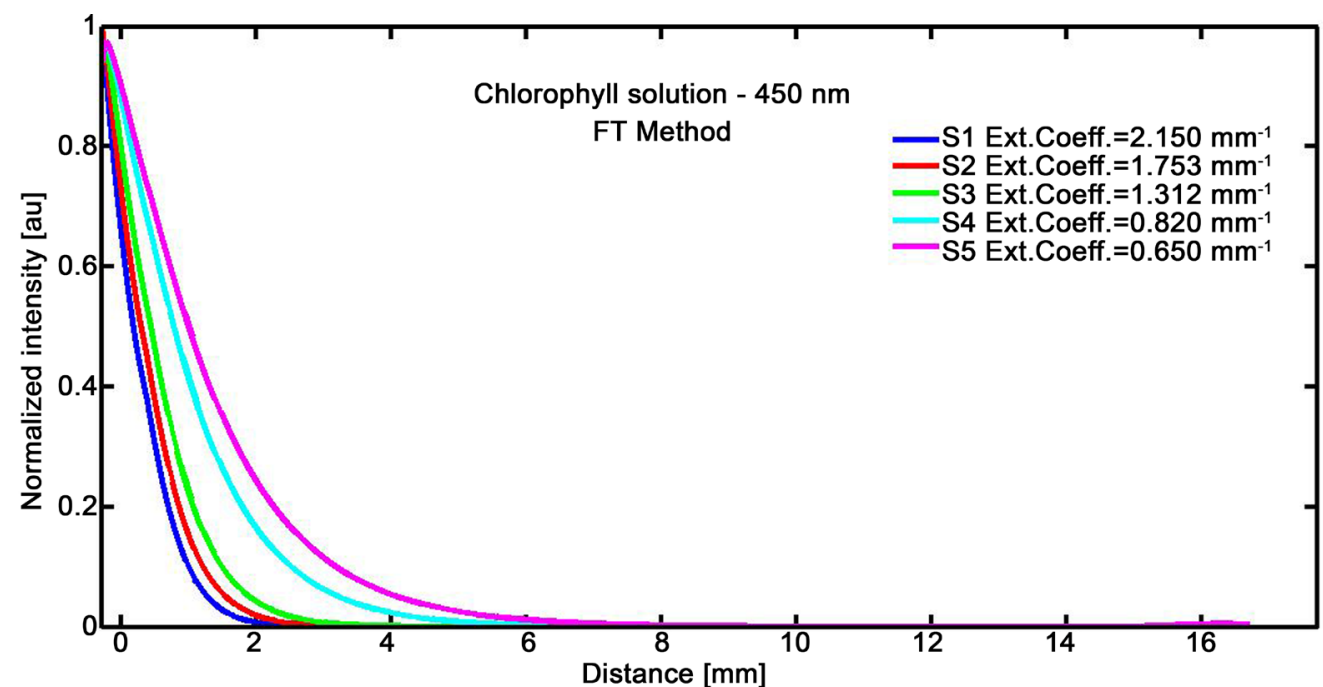

(a)

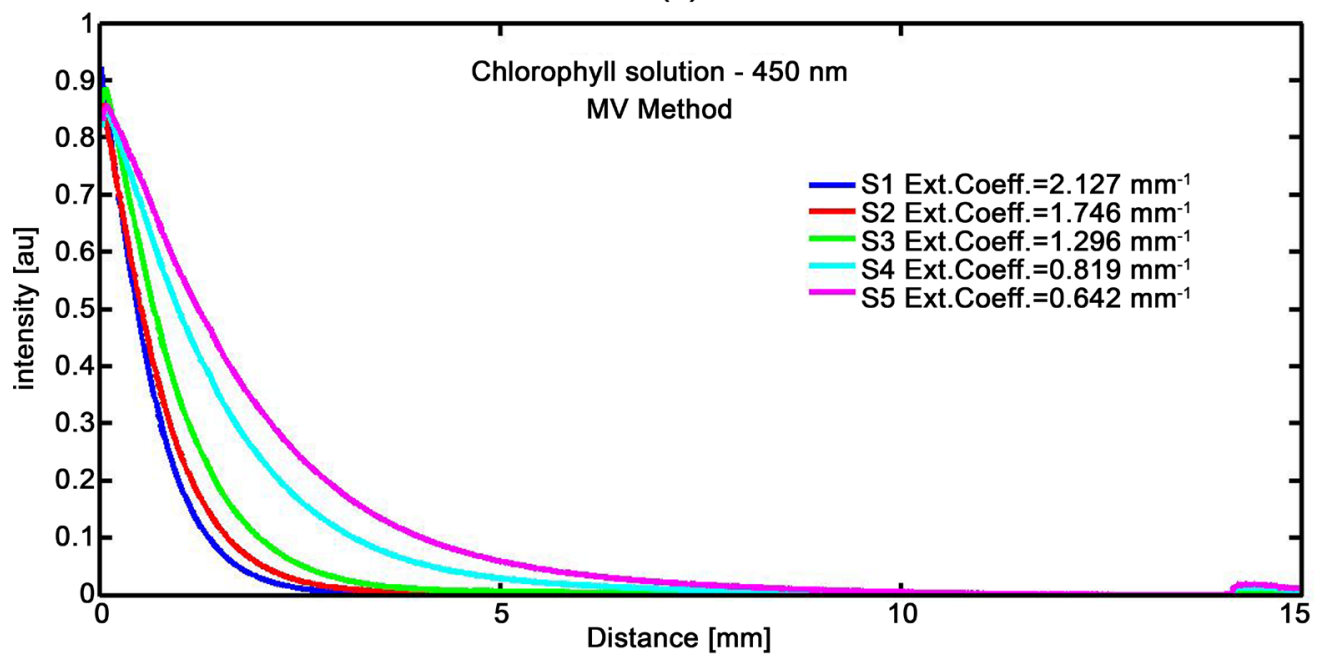

(b)

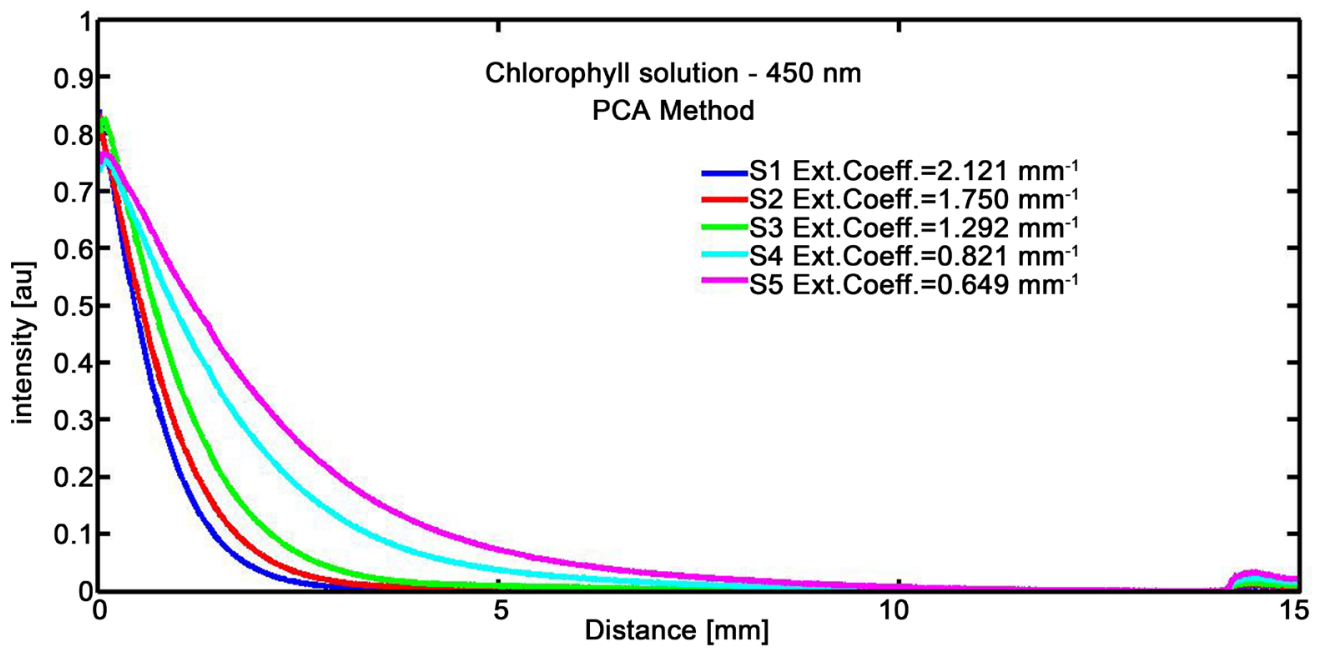

(c)

Figure 12. Curves resulting from the exponential decay of light intensity traced using each of the three methods, for the five samples of coffee solutions probed with laser light at $450 \mathrm{~nm}$. The extinction coefficients values obtained by each method are posted on each illustration. We have in top, the standard method (FT) resulting results; in the medium, those of the Mean Value (MV) method and below, the results resulting from the Principal Components Analyze (PCA) method. 


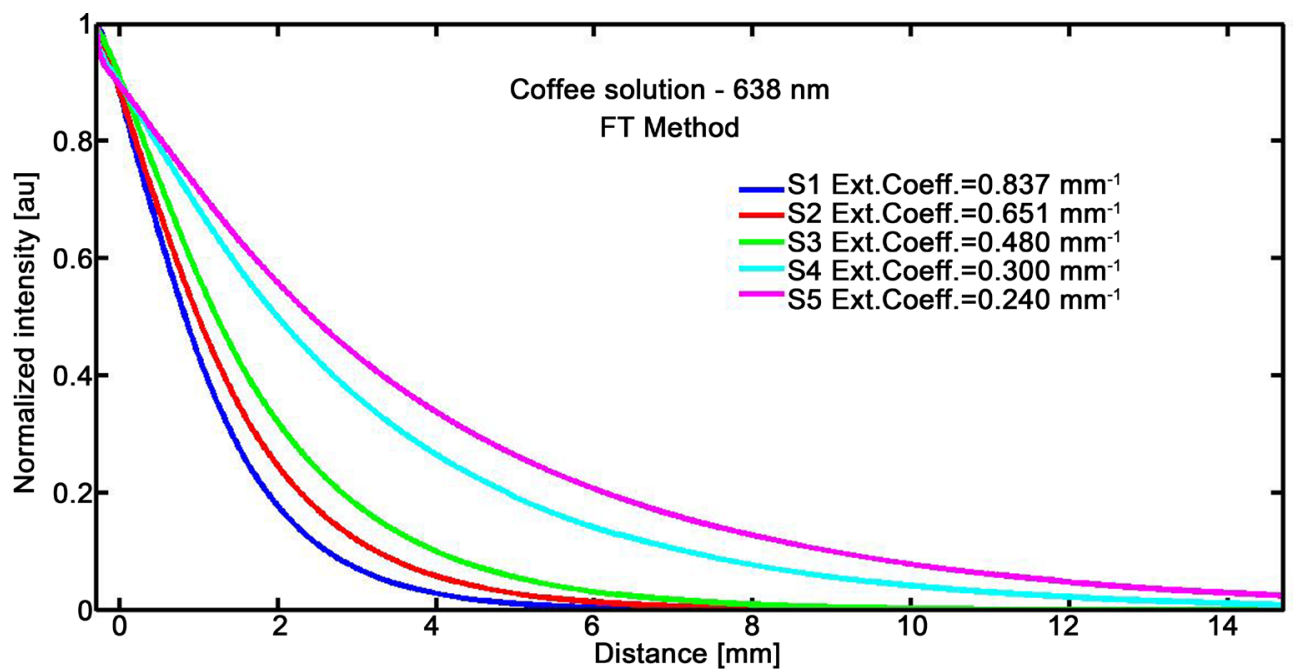

(a)

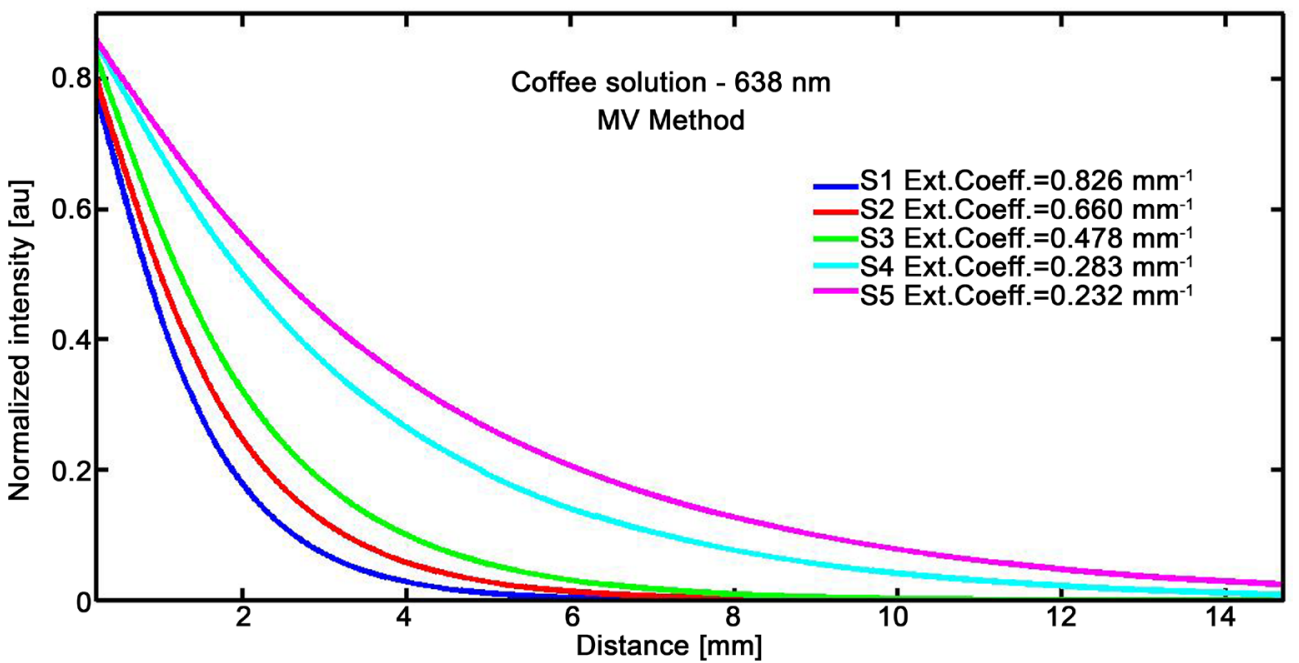

(b)

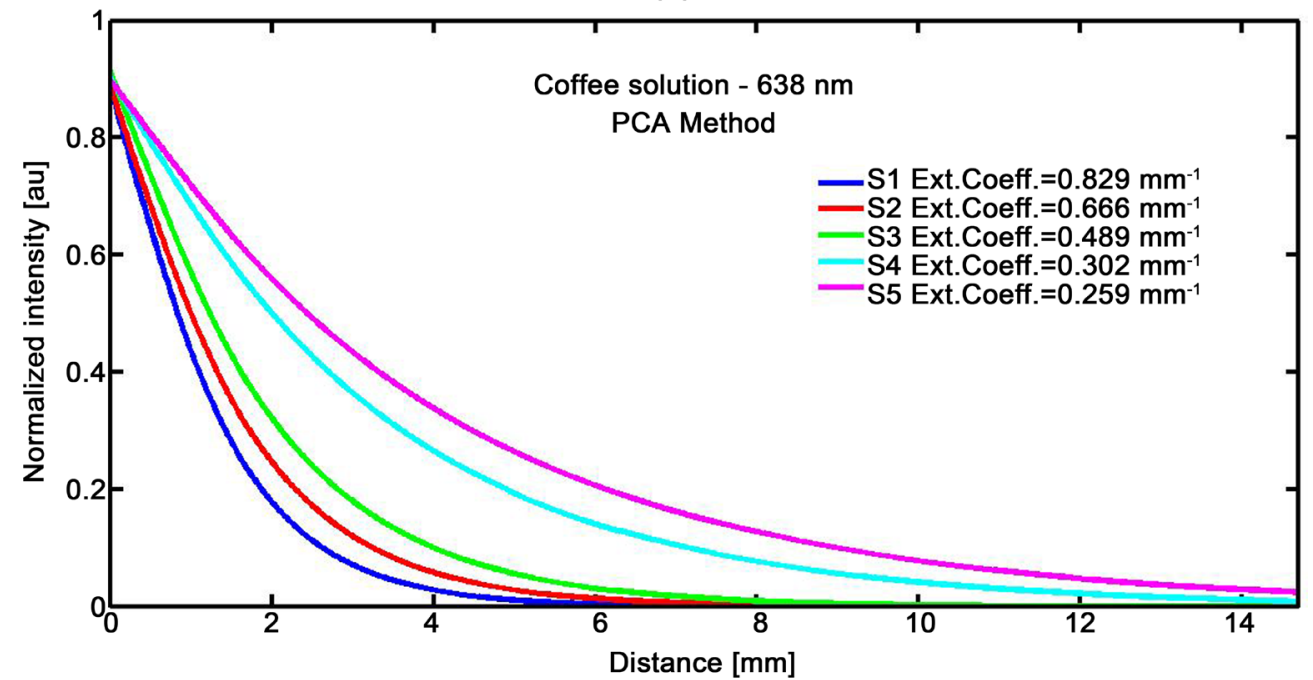

(c)

Figure 13. Curves resulting from the exponential decay of light intensity traced using each of the three methods, for the five samples of coffee solutions probed with laser light at $638 \mathrm{~nm}$. The extinction coefficients values obtained by each method are posted on each illustration. We have in top, the standard method (FT) resulting results; in the medium, those of the Mean Value (MV) method and below, the results resulting from the Principal Components Analyze (PCA) method. 
tained by each method are also nearly identical at $400 \mathrm{~nm}$ on the one hand and at $600 \mathrm{~nm}$ on the other hand.

The values of optical properties calculated via the three different approaches made it possible to trace the graphs of variability of each method according to the examined solutions concentrations. By making the differences between the extinction coefficients values obtained with the proposed methods and those of the standard method for each given sample $S i$, as Equations ((29) and (30)), we noticed very little variations.

$$
\eta_{1}=\left|\mu_{e_{s i}}(F T)_{\lambda i}-\mu_{e_{s i}}(P C A)_{\lambda i}\right|
$$

And

$$
\eta_{2}=\left|\mu_{e_{s i}}(F T)_{\lambda i}-\mu_{e_{s i}}(M V)_{\lambda i}\right|
$$

where $\mu_{e_{s i}}(F T)_{\lambda i}$ is the extinction coefficient of the sample $S i$ calculated by Fourier Transform method at the wavelength $\lambda i, \mu_{e_{s i}}(P C A)_{\lambda i}$, the extinction coefficient of the sample $S i$ calculated by PCA method at the wavelength $\lambda i$ and $\mu_{e_{s i}}(M V)_{\lambda i}$, the extinction coefficient of the sample $S i$ calculated by Mean Value method at the wavelength $\lambda i$.

Figure 14 and Figure 15 show respectively the distribution curves of these extinction coefficients variations obtained according to the Equations ((29) and (30)) with chlorophyll solutions at $450 \mathrm{~nm}$, and at $638 \mathrm{~nm}$.

Same work was also made for the extinction coefficients obtained with coffee solutions at $450 \mathrm{~nm}$ and at $638 \mathrm{~nm}$ using the three techniques.

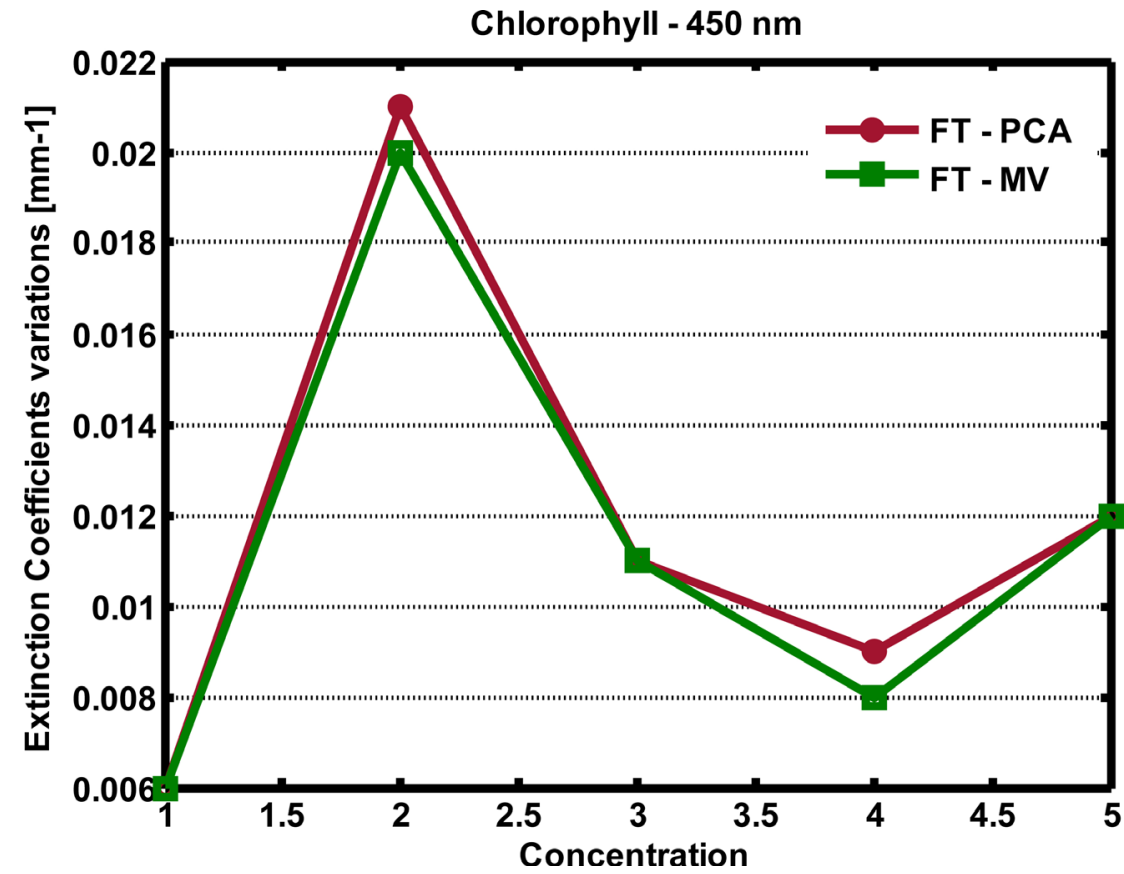

Figure 14. Variations of the extinction coefficient values calculated with FT method and those of PCA method (brown curve); then between the FT method and those of MV method (green curve) for chlorophyll solutions probed at $450 \mathrm{~nm}$. The observed deviations ranged between $0.006 \mathrm{~mm}^{-1}$ and $0.021 \mathrm{~mm}^{-1}$ for the PCA method; and these differences ranged from $0.006 \mathrm{~mm}^{-1}$ to $0.020 \mathrm{~mm}^{-1}$ for the $\mathrm{MV}$ method. 


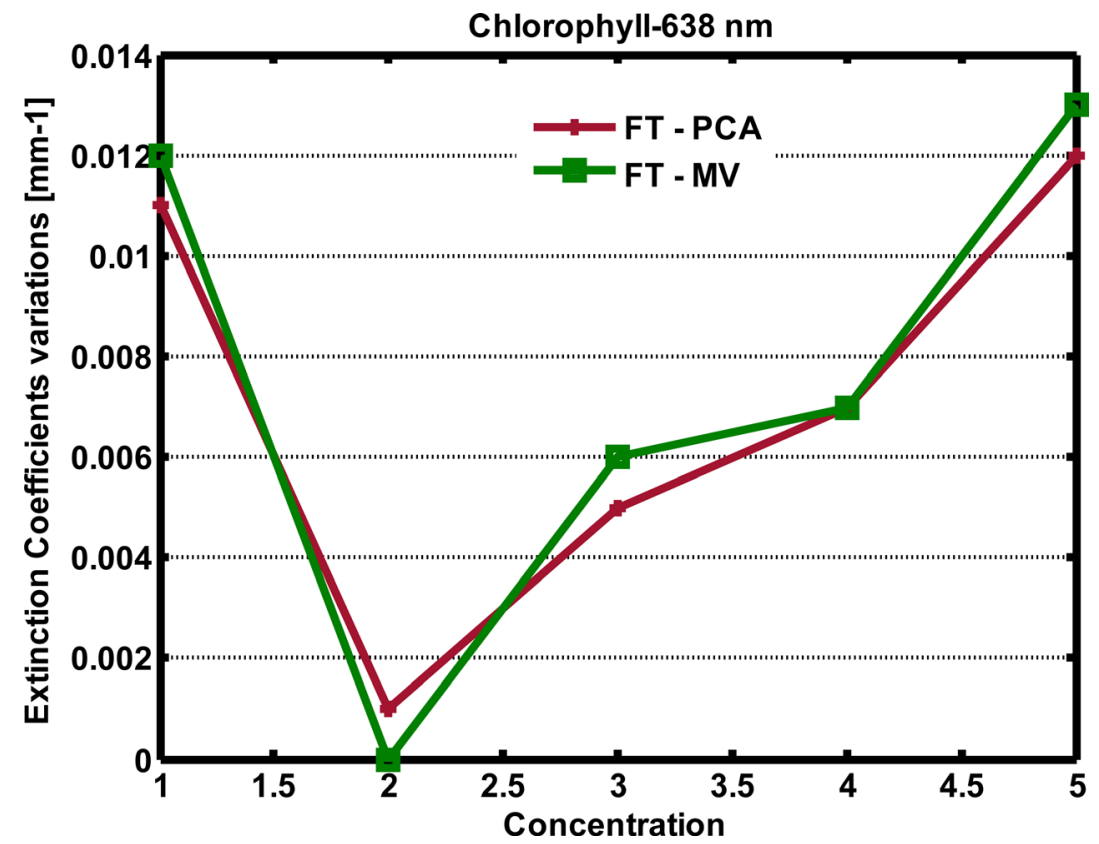

Figure 15. Variations of the extinction coefficient values calculated with FT method and those of PCA method (brown curve); then between the FT method and those of MV method (green curve) for chlorophyll solutions probed at $638 \mathrm{~nm}$. The observed deviations ranged between $0.001 \mathrm{~mm}^{-1}$ and $0.012 \mathrm{~mm}^{-1}$ for the PCA method; and these differences ranged from $0.00 \mathrm{~mm}^{-1}$ to $0.013 \mathrm{~mm}^{-1}$ for the MV method.

Note that the extinction coefficients values obtained for each type of solutions via the two proposed techniques are approximately equal to those of standard technic. However, there are small variations in these values. These are between $0.6 \%$ and $2.1 \%$ for the PCA method at $450 \mathrm{~nm}$ and between $0.1 \%$ and $1.2 \%$ for the wavelength of $638 \mathrm{~nm}$. Similarly with the Mean Value method, there are small fluctuations (slight differences) of the calculated coefficients compared to those calculated with the standard method. These small variations are estimated between $0.1 \%$ and $2.3 \%$ at $450 \mathrm{~nm}$ and between $0.0 \%$ and $0.13 \%$ for $638 \mathrm{~nm}$ radiation.

This leads to assert that these two methods that we are proposing offer similar results to the standard one.

\section{Conclusion}

The objective of this study was to provide SLIPI technique with alternative data processing methods to calculate the extinction coefficients and also optical depths of dense solutions. The objectives of this work have been achieved by the use of chlorophyll and coffee solutions at different concentration levels, and they lead satisfactory results. Note that our proposed data processing algorithms, which is based on PCA and Mean Value, made it possible to calculate extinction coefficients $\mu_{e}$ approximately equal to those obtained with the standard method [26]. Owing to the fact that they have a good computation time and are simpler concept and implementation than the standard technique, they also lead to a minimal risk of error. This approach will also be appropriate in Moiréinter- 
ferometry projection measurement. This would make possible the separation of the absorption and diffusion coefficients while interrogating dense solutions.

\section{Acknowledgements}

The authors wish to thank the International Science Program (ISP) of Uppsala University for equipment and financial support as well as the Lund Laser Center (LLC). The authors would like to thank Assoc. Prof. Edouard Berrocal for usedull discussions and valuable guidance.

\section{References}

[1] Loïc, M. (2006) Analysis and Experimental Interpretations in Mueller Polarimetry. Biomedical Applications. Ph.D. Thesis, Université de Bretagne Occidentale, Brest.

[2] Baker, W.B., Parthasarathy, A.B., Busch, D.R., Mesquita, R.C., Greenberg, J.H. and Yodh A.G. (2014) Modified Beer-Lambert Law for Blood Flow. Optical Society of America, 5, 4053-4076. https://doi.org/10.1364/BOE.5.004053

[3] Redmond, H.E., Dial, K.D. and Thompson J.E. (2010) Light Scattering and Absorption by Wind Blown Dust: Theory, Measurement, and Recent Data. Aeolian Research, 2, 5-26. https://doi.org/10.1016/j.aeolia.2009.09.002

[4] Mäntele, W. and Deniz, E. (2016) UV-VIS Absorption Spectroscopy: Lambert-Beer reloaded. Spectrochimica Acta Part A: Molecular and Biomolecular Spectroscopy, 173, 965-968.

[5] Abitan, H., Bohr, H. and Buchhave, P. (2008) Correction to the Beer-LambertBouguer Law for Optical Absorption. Applied Optics, 47, 5354-5357. https://doi.org/10.1364/AO.47.005354

[6] Sassaroli, A. and Fantini, S. (2004) Comment on the Modified Beer-Lambert Law for Scattering Media. Physics in Medicine \& Biology, 49, N255-N257.

https://doi.org/10.1088/0031-9155/49/14/n07

[7] Tam, W.G. and Zardecki, A. (1982) Multiple Scattering Corrections to the Beer-Lambert Law. Applied Optics, 21, 2405-2412. https://doi.org/10.1364/AO.21.002405

[8] LKocsis, P.H. and Eke, A. (2006) The Modified Beer-Lambert Law Revisited. Physics in Medicine and Biology, 51, 91-98. https://doi.org/10.1088/0031-9155/51/5/N02

[9] Berrocal, E. (2006) Multiple Scattering of Light in Optical Diagnostics of Dense Sprays and Other Complex Turbid Media. Ph.D. Thesis., Lund University, Sweden.

[10] Berrocal, E., Johnsson, J., Kristensson, E. and Aldén, M. (2012) Single Scattering Detection in Turbid Media Using Single-Phase Structured Illumination Filtering. Journal of the European Optical Society, 7, 6 p.

[11] Kristensson, E., Bood, J., Alden, M., Nordström, E., Zhu, J.J., Huldt, S., Bengtsson, P.-E., Nilsson, H., Berrocal, E. and Ehn, A. (2014) Stray Light Suppression in Spectroscopy Using Periodic Shadowing. Optics Express, 22, 7711-7721. https://doi.org/10.1364/OE.22.007711

[12] Kristensson, E., Berrocal, E. and Aldén, M. (2012) Quantitative 3D Imaging of Scattering Media Using Structured Illumination and Computed Tomography. Optics Express, 20, 14437-14450. https://doi.org/10.1364/OE.20.014437

[13] Kristensson, E., Berrocal, E. and Aldén, M. (2011) Extinction Coefficient Imaging of Turbid Media Using Dual Structured Laser Illumination Planar Imaging. Optics Letters, 36, 1656-1658. https://doi.org/10.1364/OL.36.001656

[14] Bagui, O.K., Kaduki, K.A., Berrocal, E. and Zoueu, J.T. (2016) Structured Laser Il- 
lumination Planar Imaging Based Classification of Ground Coffee Using Multivariate Chemometric Analysis. Applied Physics Research, 8, 32. https://doi.org/10.5539/apr.v8n3p32

[15] Neil, M.A.A., Juskaitis, R.and Wilson, T (1997) Method of Obtaining Optical Sectioning by Using Structured Light in a Conventional Microscope. Optics Letters, 22, 1905-1907. https://doi.org/10.1364/OL.22.001905

[16] Berrocal, E., Kristensson, E., Richter, M., Linne, M. and Aldén, M. (2008) Application of Structured Illumination for Multiple Scattering Suppression in Planar Laser Imaging of Dense Sprays. Optics Express, 16, 17870-17881.

https://doi.org/10.1364/OE.16.017870

https://www.osapublishing.org/oe/issue.cfm?volume=16\&issue $=22$

[17] Kristensson, E., Araneo, L., Berrocal, E., Manin, J., Richter, M., Aldén, M. and Linne, M. (2011) Analysis of Multiple Scattering Suppression Using Structured Laser Illumination Planar Imaging in Scattering and Fluorescing Media. Optics EXpress, 19, 13647-13663. https://doi.org/10.1364/OE.19.013647

[18] Kristensson, E. (2012) Structured Laser Illumination Planar Imaging: SLIPI Applications for Spray Diagnostics. Ph.D. Thesis, Lund University, Sweden.

[19] Mishra, Y.N., Kristensson, E., Pettersson, S.G. and Berrocal, E. (2013) Planar Droplets Sizing of a Hollow-Cone Spray Using SLIPI-LIF/MIE. Proceedings of the 25 th European Conference on Liquid Atomization and Spray Systems, Chania, 1-4 September 2013, 7 p.

[20] Kristensson, E., Berrocal, E. and Aldén, M. (2012) Quantitative 3D Imaging of Scattering Media Using Structured Illumination and Computed Tomography. Optics Express, 20, 14437-14450. https://doi.org/10.1364/OE.20.014437

[21] Mishra, Y.N., Kristensson, E. and Berrocal, E. (2014) Reliable LIF/Mie Droplet Sizing in Sprays Using Structured Laser Illumination Planar Imaging. Optics Express, 22, 4480-4492. https://doi.org/10.1364/OE.22.004480

[22] Kristensson, E., Richter, M., Pettersson, S.-G., Aldén, M. and Andersson-Engels, S. (2008) Spatially Resolved, Single-Ended Two-Dimensional Visualization of Gas Flow Phenomena Using Structured Illumination. Applied Optics, 47, 3927-3931. https://doi.org/10.1364/AO.47.003927

[23] Berrocal, E., Kristensson, E., Richter, M., Linne M. and Aldén, M. (2008) Multiple Scattering Suppression in Planar Laser Imaging of Dense Sprays by Means of Structured Illumination. Atomization and Sprays, 20, 133-139. https://doi.org/10.1615/AtomizSpr.v20.i2.30

[24] Kristensson, E., Ehn, A. and Berrocal, E. (2017) High Dynamic Spectroscopy Using a Digital Micromirror Device and Periodic Shadowing. Optics Express, 25, 212-222. https://doi.org/10.1364/OE.25.000212

[25] Grosshans, H., Kristensson, E., Szász, R.-Z. and Berrocal, E. (2015) Prediction and Measurement of the Local Extinction Coefficient in Sprays for 3D Simulation/Experiment Data Comparison. International Journal of Multiphase Flow, 72, 218-232. https://doi.org/10.1016/j.ijmultiphaseflow.2015.01.009

[26] Regnima, G.-O., Koffi, T., Bagui, O., Kouacou, A., Kristensson, E., Zoueu, J. and Berrocal, E. (2017) Quantitative Measurements of Turbid Liquids via Structured Laser Illumination Planar Imaging Where Absorption Spectrophotometry Fails. Applied Optics, 56, 3929-3938. https://doi.org/10.1364/AO.56.003929 
Submit or recommend next manuscript to SCIRP and we will provide best service for you:

Accepting pre-submission inquiries through Email, Facebook, LinkedIn, Twitter, etc. A wide selection of journals (inclusive of 9 subjects, more than 200 journals)

Providing 24-hour high-quality service

User-friendly online submission system

Fair and swift peer-review system

Efficient typesetting and proofreading procedure

Display of the result of downloads and visits, as well as the number of cited articles Maximum dissemination of your research work

Submit your manuscript at: http://papersubmission.scirp.org/

Or contact ojapps@scirp.org 